\title{
Effect of Particle Size and Doping on the Electrochemical Characteristics of Ca-doped $\mathrm{LiCoO}_{2}$ Cathodes
}

Fuead Hasan, Jinhong Kim, Heewon Song, Seon Hwa Lee, Jong Hun Sung, Jisu Kim, and Hyun Deog Yoo* Department of Chemistry and Chemical Institute for Functional Materials, Pusan National University, Busan 46241, Korea

\begin{abstract}
Lithium cobalt oxide $\left(\mathrm{LiCoO}_{2}, \mathrm{LCO}\right)$ has been widely used as a cathode material for Li-ion batteries (LIBs) owing to its excellent electrochemical performance and highly reproducible synthesis even with mass production. To improve the energy density of the LIBs for their deployment in electro-mobility, the full capacity and voltage of the cathode materials need to exploited, especially by operating them at a higher voltage. Herein, we doped LCO with divalent calcium-ion $\left(\mathrm{Ca}^{2+}\right)$ to stabilize its layered structure during the batteries' operation. The Ca-doped LCO was synthesized by two different routes, namely solid-state and co-precipitation methods, which led to different average particle sizes and levels of dopant's homogeneity. Of these two, the solid-state synthesis resulted in smaller particles with a better homogeneity of the dopant, which led to better electrochemical performance, specifically when operated at a high voltage of $4.5 \mathrm{~V}$. Electrochemical simulations based on a single particle model provided theoretical corroboration for the positive effects of the reduced particle size on the higher rate capability.
\end{abstract}

Keywords : Lithium-Ion Batteries, Lithium Cobalt Oxide, Particle Size, Solid-State Synthesis, Co-Precipitation

Received : 1 April 2020, Accepted : 11 May 2020

\section{Introduction}

The application and storage of renewable energy is needed to cope with unpredictable climate changes and depletion of natural resources. To this end, development of power generation devices that are immune to environmental hazards have been actively investigated [1]. Advancement of power storage technologies, such as Li-ion batteries (LIBs), redox flow batteries, and metal-air batteries, have demonstrated noticeable progress. Among them, LIBs have evolved from portable electronics to electric vehicles (EVs) and energy storage systems (ESSs). The increasing concerns on climate change has led to stricter stipulations for electro-mobility, which demand rechargeable batteries with higher energy densities [2]. Advanced cathode materials are essential to develop LIBs with higher performances. As a result, the battery industry has witnessed various

*E-mail address: hyundeog.yoo@pusan.ac.kr DOI: https://doi.org/10.33961/jecst.2020.00899

This is an open-access article distributed under the terms of the Creative Commons Attribution Non-Commercial License (http://creativecommons.org/licenses/by-nc/4.0) Attribution Non-Commercial License (http://creativecommons.org/licenses/by-nc/4.0)
which permits unrestricted non-commercial use, distribution, and reproduction in any which permits unrestricted non-commercial use, distri
medium, provided the original work is properly cited. cathode materials over the past 30 years, even though only a few of them were successfully commercialized. Voltage and capacity fading, structural and thermodynamic instability, and poor ionic conductivity have been identified as the barriers for the development of advanced cathode materials [3,4]. On the other hand, lithium cobalt oxide $\left(\mathrm{LiCoO}_{2}, \mathrm{LCO}\right)$, first identified as a cathode material by Goodenough et al. [5], exhibits a high theoretical capacity and moderate $\mathrm{Li}^{+} /$electron conductivity, which are beneficial for a reasonable electrochemical performance [6-8]. In addition, the legendary $\mathrm{LiCoO}_{2}$ or LCO is still actively utilized as an active cathode material in LIBs owing to its highly reproducible synthesis and superior electrochemical performance $[9,10]$. For this reason, it might be meaningful to revisit the LCO cathodes for a comprehensive exploitation of their theoretical electrochemical performance.

Even though the theoretical capacity of LCO is as high as $274 \mathrm{mAh} \mathrm{g}^{-1}$, only up to $60 \%$ of this capacity has been practically utilized, which is approximately $165 \mathrm{mAh} \mathrm{g}^{-1}$ [11]. When compared to $\mathrm{Li} / \mathrm{Li}^{+}, \mathrm{LiCoO}_{2}$ experiences order-disorder phase transition, structural instability, and severe capacity loss when it is 
operated at more than $4.3 \mathrm{~V}$. Charging up to $4.2 \mathrm{~V}$ results in an order-disorder transition from hexagonal to monoclinic structure, as approximately half of the $\mathrm{Li}^{+}$ions are dislodged from the host structure. Upon further charging above $4.5 \mathrm{~V}$, another transition occurs from $\mathrm{O} 3$ to H1-3 or O6 phases, caused by various physio-chemical interactions inside the cathode [12-15]. The order-disorder transitions extensively lower the $\mathrm{Li}^{+}$diffusivity, and the transition to the $\mathrm{H} 1$ 3 phase critically destroys the structure of the cathode material despite the completely reversible phase transitions. Therefore, it is very essential to enhance the LCO cathodes in terms of the cutoff voltage and fast chargeability $[16,17]$. To this end, doping LCO with various metal ions has resulted in various degrees of success $[18,19]$. The incorporation of divalent ions into the $\mathrm{LiO}_{2}$-layer can stabilize the structure [20]; moreover, such divalent dopants can prevent cation mixing by compensating for the deficiency of $\mathrm{Li}^{+}$ ions in layered materials $[21,22]$. Among the divalent dopants, $\mathrm{Ca}^{2+}$ has been proposed as one of the effective dopants for improved cycling stability. Various methods have been developed for ion doping, such as co-precipitation, solid-state, sol-gel, molten salt, and hydrothermal methods. Among these, solid state and co-precipitation methods have been actively utilized owing to their low cost and scalability.

In theory, a small particle size is beneficial to enhance the rate capability of cathode materials $[23,24]$. The reduced particle size shortens the diffusion pathways for $\mathrm{Li}^{+}$ions, thus creating a large contact area between the active materials and the conductive additives [25]. The rate determining step of the discharging process at a high rate operation contributes to the solid-state diffusion of $\mathrm{Li}^{+}$ions inside the electrodes [26,27]. The beneficial effects of reduced particle size include structural durability as a result of the lower concentration gradient developed inside the particles. High-energy ball milling has been a reliable process to reduce the particle size of cathode materials to sub-micron scales [28,29]. On the other hand, it has been reported that excessive nano-sizing is detrimental to the electrochemical performance of cathode materials [30,31], because it promotes side reactions at high voltages and leads to structural damage. Therefore, it is important to investigate the effect of particle size on the electrochemical performance of cathode materials that are prepared by various synthetic routes.
Herein, we report a comparative study on the electrochemical performance of Ca-doped $\mathrm{LCO}\left(\mathrm{LiCo}_{1-x}\right.$ $\mathrm{Ca}_{x} \mathrm{O}_{2}, x=0.02$ ) that were synthesized by two different routes, namely solid-state synthesis and co-precipitation. The solid-state synthesis of Ca-doped LCO resulted in a smaller particle size and better homogeneity of the dopants compared with the coprecipitated sample. This would result in a higher cycling stability and rate capability. Electrochemical simulations using various particle sizes supported the effective role of reduced particle size on the rate capability. These results have demonstrated the pivotal importance of reducing the particle size and homogeneous doping to improve the electrochemical performance of layered cathode materials, especially at higher operational voltages.

\section{Experimental}

\subsection{Solid-state synthesis of Ca-doped LCO}

For the solid-state synthesis of Ca-doped LCO, stoichiometric amounts of lithium carbonate $\left(\mathrm{Li}_{2-}\right.$ $\mathrm{CO}_{3}$; Junsei Chemical Co., Japan; 99\%), cobalt oxide black $\left(\mathrm{Co}_{3} \mathrm{O}_{4}\right.$; Junsei Chemical Co., Japan; $\left.>85 \%\right)$, and calcium nitrate tetrahydrate $\left(\mathrm{Ca}\left(\mathrm{NO}_{3}\right)_{2} \cdot 4 \mathrm{H}_{2} \mathrm{O}\right.$; Kanto Chemicals Co., Japan; 98.5\%) were ball milled, calcined, and sintered. Essentially, $4.02 \mathrm{~g}$ of $\mathrm{Li}_{2} \mathrm{CO}_{3}, 9.49 \mathrm{~g}$ of $\mathrm{Co}_{3} \mathrm{O}_{4}$, and $0.492 \mathrm{~g}$ of $\mathrm{Ca}\left(\mathrm{NO}_{3}\right)_{2} \cdot 4 \mathrm{H}_{2} \mathrm{O}$ were initially mixed in an agate mortar and pestle, then thoroughly mixed and ground in a planetary ball mill (PM 100, Retsch Co., Germany) at $3000 \mathrm{rpm}$ for $4 \mathrm{~h}$. The mechano-chemically homogenized precursors were then calcined at $800^{\circ} \mathrm{C}$ for $12 \mathrm{~h}$ and cooled down naturally. Thus, the initially calcined powder was ground and mixed well before final sintering at $850^{\circ} \mathrm{C}$ for $24 \mathrm{~h}$. All the calcination and sintering were carried out in an air atmosphere. The resultant material was labeled as SS-Ca-LCO, where SS denotes solid-state synthesis. For comparison, pristine LCO without doping was synthesized as well by solid-state route and labeled as SS-PLCO.

\subsection{Co-precipitation of Ca-doped LCO}

For comparison, a precursor for Ca-doped LCO was synthesized by co-precipitation of $\mathrm{Co}_{0.98}$ $\mathrm{Ca}_{0.02}(\mathrm{OH})_{2}$. Aqueous solution of cobalt nitrate hexahydrate $\left(\mathrm{Co}\left(\mathrm{NO}_{3}\right)_{2} \cdot 6 \mathrm{H}_{2} \mathrm{O}\right.$; Junsei Chemicals Co., Japan; $98.0 \%)$ and $\mathrm{Ca}\left(\mathrm{NO}_{3}\right)_{2} \cdot 4 \mathrm{H}_{2} \mathrm{O}$ was prepared according to stoichiometry. Aqueous ammonia 
$\left(\mathrm{NH}_{3} \cdot \mathrm{H}_{2} \mathrm{O}\right.$; Daejung Chemicals \& Metals Co., Korea; $25-28 \%$ ) was added to the solution of metal salts, so that the concentrations of the metal ions and $\mathrm{NH}_{3}$ were 2.0 and $1.0 \mathrm{M}$, respectively, and the total volume of the solution was $100 \mathrm{~mL}$. Separately, $1 \mathrm{M}$ $\mathrm{NH}_{3}$ aqueous solution of $1 \mathrm{~L}$ was prepared and filled in the reactor beforehand. Subsequently, the metal and $\mathrm{NH}_{3}$ solution was added dropwise using a peristaltic pump (Multi-channel Pump, JiPump Co.; China) at a speed of $0.04 \mathrm{~L} \mathrm{~h}^{-1}$ under an inert atmosphere of $\mathrm{N}_{2}$. Here, ammonia was employed as an auxiliary complexing agent, so that the precipitation of metal ions are retarded for more homogeneous composition of the particles [32]. During the co-precipitation reaction, the solution was vigorously agitated using an overhead stirrer at $350 \mathrm{rpm}$, with the temperature set to $60^{\circ} \mathrm{C}$ using a heating mantle. Simultaneously, a $4 \mathrm{M}$ sodium hydroxide $(\mathrm{NaOH}$; Yakuri Pure Chemicals Co., 96.0\%) solution was added dropwise using a computerized peristaltic pump (Masterflex L/S computer-compatible drive, 07551-30 series, Cole-Parmer Co; USA) to maintain the $\mathrm{pH}$ value at 10.50 during the co-precipitation reaction. After $1 \mathrm{~h}$ of co-precipitation, the reactor was cooled down to ambient temperature and the precipitated solution was centrifuged for $10 \mathrm{~min}$ at 3000 $\mathrm{rpm}$. Subsequently, the supernatant was carefully discarded and the precipitate was mixed with fresh deionized water. The centrifuging was repeated two more times. The precipitate was rinsed with deionized water several times and filtered before being dried for $2 \mathrm{~h}$ at $80^{\circ} \mathrm{C}$ in a vacuum oven. The resultant light pinkish-brown powder of $\mathrm{Co}_{0.98} \mathrm{Ca}_{0.02}(\mathrm{OH})_{2}$ was mixed with a stoichiometric amount of lithium hydroxide monohydrate $\left(\mathrm{LiOH} \cdot \mathrm{H}_{2} \mathrm{O}\right.$; Junsei Chemicals Co., 98\%) using an agate mortar and a pestle. The mixed powder was heated in a box furnace for $12 \mathrm{~h}$ at $800^{\circ} \mathrm{C}$, at a heating rate of $100^{\circ} \mathrm{C} / \mathrm{h}^{-1}$ and a natural cooling rate. The calcined powder was ground and mixed well before finally being sintered at $850^{\circ} \mathrm{C}$ for $24 \mathrm{~h}$. The Ca-doped lithium cobalt oxide $\left(\mathrm{LiCo}_{0.98} \mathrm{Ca}_{0.02} \mathrm{O}_{2}\right.$ ) powder thus obtained, was ground in an agate mortar before the characterization. The resultant material was labeled as $\mathrm{CP}-\mathrm{Ca}-\mathrm{LCO}$, where $\mathrm{CP}$ denotes co-precipitation.

\subsection{Materials Characterization}

For structural analysis, X-ray diffractometry (XRD; MiniFlex 600, Rigaku Co., Japan) was per- formed in a range of $10-80^{\circ}(2 \theta)$ with a step width of $0.02^{\circ}$ and scanning speed of $10^{\circ} \mathrm{min}^{-1}$. The particles were imaged using a scanning electron microscope (SEM; VEGA3, Tescan Co. and S3500, Hitachi Co.: Japan) and a scanning transmission electron microscope (STEM; Talos F200X, Thermo Fisher Scientific Co.; USA) The homogeneity of dopants in Cadoped LCO particles and other elemental information was obtained using energy dispersive spectroscopes (EDS) that were attached to the SEM and TEM instruments.

\subsection{Electrochemical Characterization}

The electrodes were prepared by coating a slurry of N-methyl-2-pyrrolidone (NMP; Sigma Aldrich, USA; 99.5\%) that is mixed with Ca-doped LCO, conductive carbon black (Super-P, Timcal Co.), and polyvinylidene fluoride (PVDF; KF1100, Kureha Co.) in a mass ratio of $6: 2: 2$ on an aluminum foil of $20 \mathrm{~mm}$ using a doctor blade. The coated electrode was dried at $80^{\circ} \mathrm{C}$ under vacuum for $2 \mathrm{~h}$. Next, the dried electrode was roll-pressed and punched into discs of $0.95 \mathrm{~cm}^{2}$ with an active material loading of 1-2 mg. Coin cells of 2032-type were assembled with the coated electrode as the cathode and a lithium foil as the anode, both of which sandwiched a separator that was soaked with an electrolyte (Panax-Etec Co.; Korea) of $1 \mathrm{M}$ lithiumhexafluorophosphate $\left(\mathrm{LiPF}_{6}\right)$ in ethylene carbonate and ethyl-methyl-carbonate (EC:EMC, 3:7 w/ w) inside a glove box (Moisture Oxygen Technology (MOTEK) Co.; Korea), where the moisture and oxygen levels were kept below 1 ppm.

\subsection{Electrochemical Simulation}

A one-dimensional single particle model (SPM) was assumed to simulate a battery system that includes the $\mathrm{LiCoO}_{2}$ cathode, the electrolyte, and the $\mathrm{Li}$ metal anode. The open circuit potential was determined as a function of intercalation quantity (i.e., $x$ in $\mathrm{Li}_{x} \mathrm{CoO}_{2}$ ) by galvanostatic intermittent titration technique (GITT). The electrochemical (de)intercalation at the electrode-electrolyte interface was described by Fick's first law and the Butler-Volmer equation; and the solid-state chemical diffusion of Li-ions in the electrode particle was modeled by Fick's second law. Exchange current density for the (de)intercalation of $\mathrm{Li}^{+}$ions was set to $0.5 \mathrm{~mA} \mathrm{~cm}^{-2}$ for all the simulations in this work. Conductivity, transference number, and thickness of the electrolyte were set to 
$10 \mathrm{mS} \mathrm{cm}{ }^{-1}, 0.3$, and $20 \mu \mathrm{m}$, respectively, which are close to the specification of commercial electrolytes for LIBs. The conductivity of the electrolyte was assumed to be a function of concentration according to Kohlrausch's law. Using this SPM, batteries' electrochemical performances were simulated with respect to the LCO's particle size, C-rate, and solidstate diffusivity. The time-dependent partial differential equations in weak formulations were numerically solved by finite element method (FEM) using COMSOL Multiphysics 5.5 software at Precision 7920 workstation (DELL Co.) with 24 cores.

\section{Results and Discussion}

Figs. 1(a) and 1(b) show the SEM images of $\mathrm{LiCo}_{0.98} \mathrm{Ca}_{0.02} \mathrm{O}_{2}$ powders that were synthesized by solid-state (SS-Ca-LCO) and co-precipitation (CP$\mathrm{Ca}-\mathrm{LCO}$ ) routes, respectively. SS-Ca-LCO exhibited smaller and more uniform particle size compared to CP-Ca-LCO. The morphology particle size of SSPLCO were similar to that of SS-Ca-LCO (Fig. S1). The average particle sizes (i.e., diameter, $d$ ) were approximately 1.5 and $3.8 \mu \mathrm{m}$ for SS-Ca-LCO and
CP-Ca-LCO, respectively. This signifies that the mechano-chemical route (e.g., high-energy ball milling) could provide a smaller and more evenly distributed particle size distribution with less agglomeration compared to wet-chemical routes, such as co-precipitation. The nucleation and growth of the coprecipitated precursors are determined by various factors such as feeding rate, concentration of solutions, stirring rate, and reactor volume [33]. We speculate that the growth of the precursors were particularly preferred by the experimental condition of coprecipitation in this work. Another possible factor for the larger particle size of CP-Ca-LCO is the manual grinding with mortar and pestle. Fig. 1(c) shows the XRD patterns of Ca-doped LCO from the two different synthetic routes compared with that of pristine LCO from solid-state synthesis. The diffraction patterns of Ca-doped LCO matched with the pattern of pure $\mathrm{LiCoO}_{2}$, which was a hexagonal structure of layered sodium ferrite $\left(\alpha-\mathrm{NaFeO}_{2}\right)$ with the space group of $R-3 m$. The structural information was further determined using Rietveld refinement, as shown in Fig. 1(d) and Table 1. The $c / a$ values of the all the prepared samples were found to be more than 4.99 , (a)
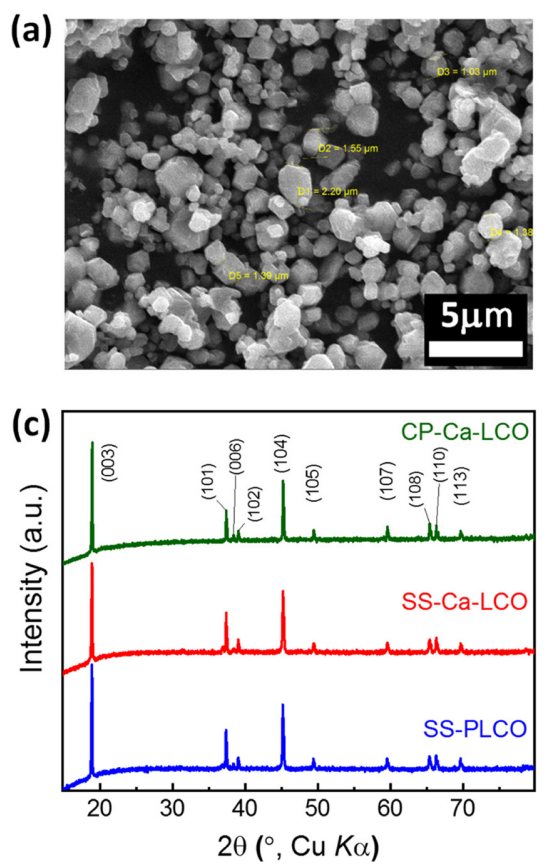

(b)
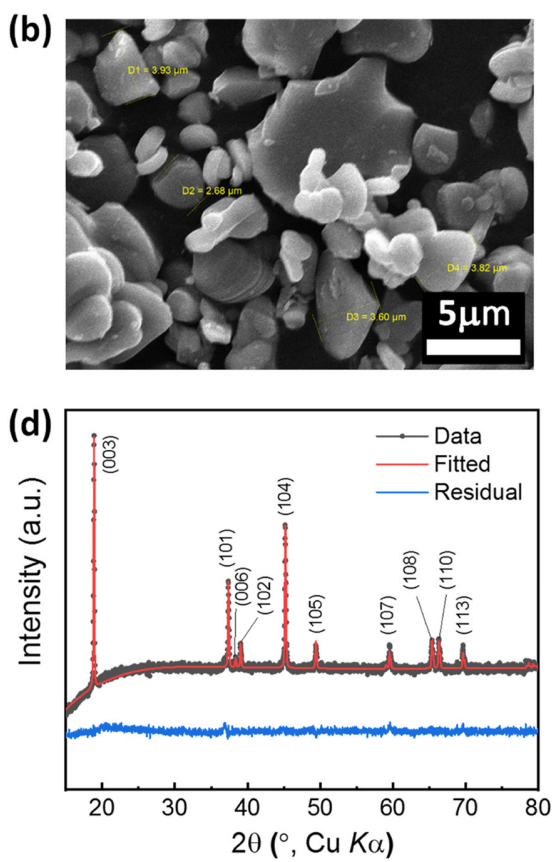

Fig. 1. SEM images of Ca-doped LCO prepared by two different synthetic routes: (a) solid-state synthesis (SS-Ca-LCO) and (b) co-precipitation (CP-Ca-LCO); (c) XRD patterns of pristine and Ca-doped LCO samples; and (d) the Rietveld refinement of SS-Ca-LCO. 
Table 1. Rietveld refinement of pristine and Ca-doped LCOs

\begin{tabular}{ccccccc}
\hline \hline Sample & $a(\AA)$ & $c(\AA)$ & $c / a$ & $I(003) / I(104)$ & $R_{\text {wp }}(\%)$ & $S$ \\
\hline SS-PLCO & 2.8160 & 14.0626 & 4.9938 & 1.272 & 1.74 & 1.23 \\
SS-Ca-LCO & 2.8171 & 14.0678 & 4.9937 & 1.272 & 0.94 & 1.38 \\
CP-Ca-LCO & 2.8162 & 14.0567 & 4.9913 & 1.49 & 2.21 & 3.48 \\
\hline
\end{tabular}

(a)
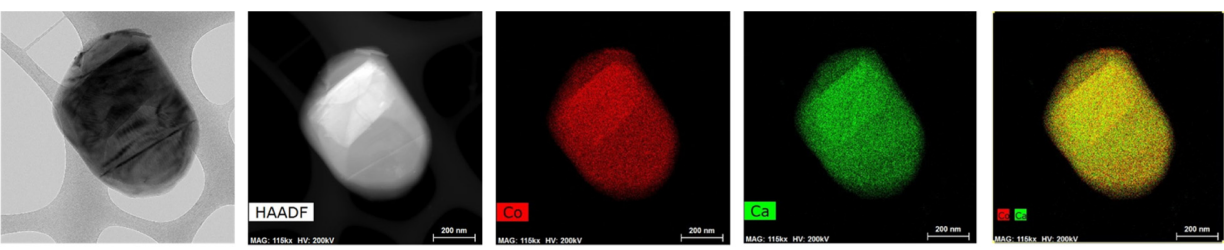

(b)

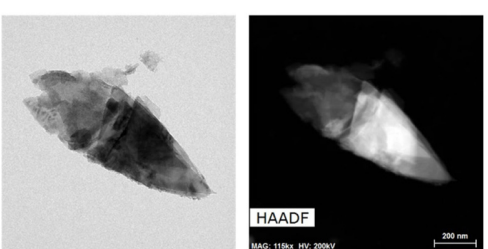

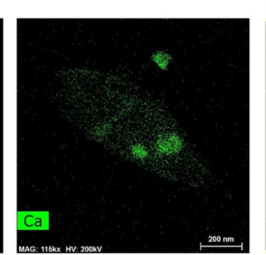

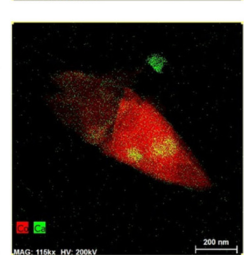

Fig. 2. STEM images in bright and dark field modes, together with the elemental maps for SS-Ca-LCO (a) and CP-Ca$\mathrm{LCO}(\mathrm{b})$.

which were higher than the critical value of 4.9; any value lower than the critical value signifies the development of a disorderly rock salt structure. $I(003) /$ $I(104)$, the ratio of intensity for (003) plane to that for (104) plane, has been utilized as a useful criteria to determine the degree of "cation mixing," which is the exchange of $\mathrm{Li}^{+}$and transition metal ions that is detrimental to the electrochemical performances [34]. $I(003) / I(104)$ ratio that is lower than 1.2 indicates a significant degree of cation exchange. The $I(003) /$ $I(104)$ ratio was larger than 1.2 for all the samples, indicating that cation mixing did not occur.

Fig. 2 shows the STEM images in bright and darkfield modes together with elemental maps for $\mathrm{Ca}-$ doped LCO. Among them, SS-Ca-LCO represented more homogeneous distribution of $\mathrm{Ca}$ compared to CP-Ca-LCO. The less homogenous distribution of $\mathrm{Ca}^{2+}$ ions in the CP-Ca-LCO sample may be deeply related with the non-homogenous coprecipitaiton of $\mathrm{Ca}^{2+}$ ions in the hydroxide precursor. The solubility product $\left(K_{\mathrm{sp}}\right)$ of $\mathrm{Ca}(\mathrm{OH})_{2}$ is $6.5 \times 10^{-6}$, which is significantly larger than $1.3 \times 10^{-15}$ for $\mathrm{Co}(\mathrm{OH})_{2}$; this means that the precipitation of $\mathrm{Co}(\mathrm{OH})_{2}$ can be kinetically much more preferred compared to $\mathrm{Ca}(\mathrm{OH})_{2}$. The different kinetics of precipitation for $\mathrm{Co}^{2+}$ and $\mathrm{Ca}^{2+}$ may have led to the non-homogeneous agglom- eration. For such cases, solid-state mechanochemical doping can be more beneficial for homogeneous distribution of dopants. EDS analysis provided evidence that the proportion of calcium $(x)$ in $\mathrm{LiCo}_{1-x} \mathrm{Ca}_{x} \mathrm{O}_{2}$ was approximately 0.019 and 0.017 for SS-Ca-LCO and CP-Ca-LCO, respectively (Fig. S2). These values were close to the targeted value of 0.02 .

Fig. 3(a) shows the electrochemical charge/discharge profiles of SS-PLCO, SS-Ca-LCO, and CP$\mathrm{Ca}-\mathrm{LCO}$ in the potential range of 3.0 and $4.5 \mathrm{~V}$ vs $\mathrm{Li} /$ $\mathrm{Li}^{+}$at $0.1 \mathrm{C}$ rate (i.e., $1 \mathrm{C}=274.89 \mathrm{~mA} \mathrm{~g}^{-1}$, corresponding to the theoretical capacity of LCO). The initial capacity was $180 \mathrm{mAh} \mathrm{g}^{-1}$ and $169 \mathrm{mAh} \mathrm{g}^{-1}$ for Ca-doped LCOs and pristine LCO, respectively, evidencing the enhanced electrochemical performance of Ca-doped LCOs. Profiles of both Ca-doped LCOs coincided significantly with each other, with an initial discharge capacity of approximately $180 \mathrm{mAh}$ $\mathrm{g}^{-1}$. This signifies that both the samples shared similar material properties as electrode materials. The cycle test at $0.1 \mathrm{C}$ in the range of $3.0-4.5 \mathrm{~V}$ vs $\mathrm{Li} / \mathrm{Li}^{+}$ yielded a higher capacity retention of $94.2 \%$ for SSCa-LCO compared to $91.1 \%$ for CP-Ca-LCO after 25 cycles. On the other hand, SS-PLCO exhibited continually lower capacity compared to the Ca-doped LCOs throughout the cycling. Moreover, the long- 
(a)

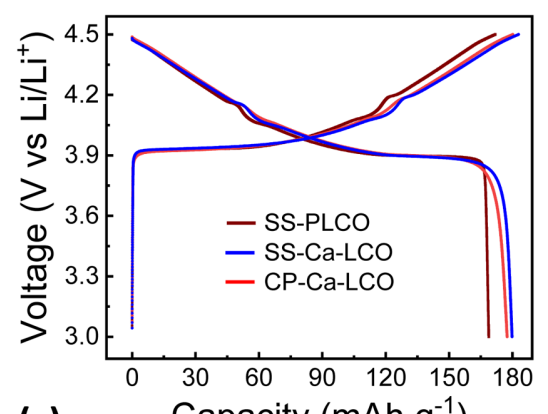

(c) Capacity $\left(\mathrm{mAh} \mathrm{g}^{-1}\right)$

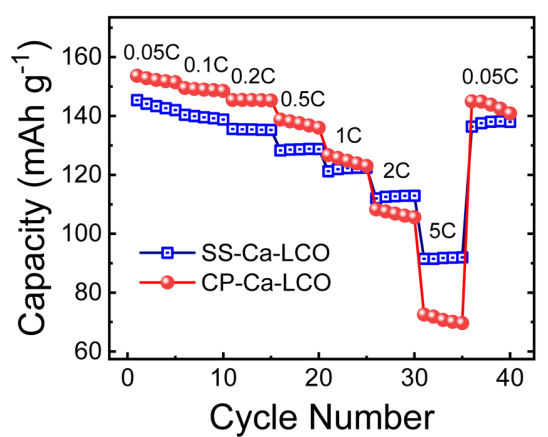

(b)
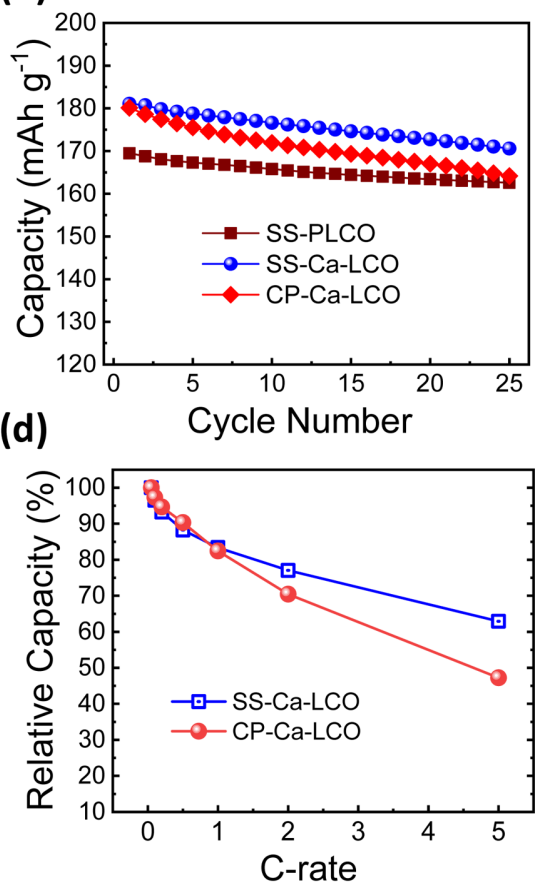

Fig. 3. Electrochemical performance of pristine and Ca-doped LCOs: (a) charge-discharge profiles; (b) cycle performance; (c) rate performance; and (d) relative capacity represented as a function of C-rate based on the results from (c). Note that the electrochemical tests were conducted in the range of $3.0-4.5 \mathrm{~V}$ vs $\mathrm{Li} / \mathrm{Li}^{+}$at $0.1 \mathrm{C}$, except for the rate capability tests in (c) and (d), which were conducted in the range of $3.0-4.3 \mathrm{~V} \mathrm{vs} \mathrm{Li} / \mathrm{Li}^{+}$to prevent electrolyte decomposition during the rate test.

term cycling of 100 cycles at accelerated rate of $0.5 \mathrm{C}$ exhibited higher cycleability of Ca-doped LCO compared to pristine LCO (Fig. S3), evidencing the enhanced structural stability by Ca-doping. SS-CaLCO also exhibited better rate capability compared to CP-Ca-LCO as shown in Figs. 3(c) and 3(d). The specific capacity at $5 \mathrm{C}$ (i.e., charging or discharging batteries in $12 \mathrm{~min}$ ) was 91.95 and $69.64 \mathrm{mAhg}^{-1}$ for SS-Ca-LCO and CP-Ca-LCO, respectively; these values correspond to $62.9 \%$ and $47.2 \%$ retention with respect to the initial capacity values.

The better cycle and rate performances of SS-CaLCO compared to CP-Ca-LCO could be ascribed to the following factors: (1) smaller particle size and (2) more homogeneous distribution of $\mathrm{Ca}^{2+}$ dopants. Smaller particle size enabled a decrease in the pathway for ionic diffusion $(l)$, thus reducing the time constant for ionic diffusion according the relationship $t \sim l^{2} / D$; here the solid-state chemical diffusion coefficient $(D)$ is a constant. The reduced time constant signifies a faster diffusion in the cathode materials, which would enable a higher rate capability. Moreover, a faster transport of ions in smaller particle size resulted in a smaller concentration gradient $(\mathrm{d} c / \mathrm{d} r)$; here $c$ denotes the $\mathrm{Li}^{+}$concentration as a function of the radial distance $(r)$ from the center of a particle. As the concentration gradient might result in a mechanical stress, a smaller particle size could minimize the mechanical stress and provide a better cycle performance. On the other hand, a better homogeneity of $\mathrm{Ca}^{2+}$ ions within LCO layers could strengthen the structure against the insertion/extraction processes during the batteries' operation. These manifold effects may have resulted in a better rate and cycle performances of SS-Ca-LCO compared to those of CP-Ca-LCO.

The discharge voltage profiles of SS-Ca-LCO and CP-Ca-LCO at different C-rates are shown in Figs. 4(a) and 4(b), respectively. Not only the capacity but also the average voltage decreased with the C-rate, implying a more significant reduction in the delivered energy. It was clearly observed that SS-Ca-LCO 

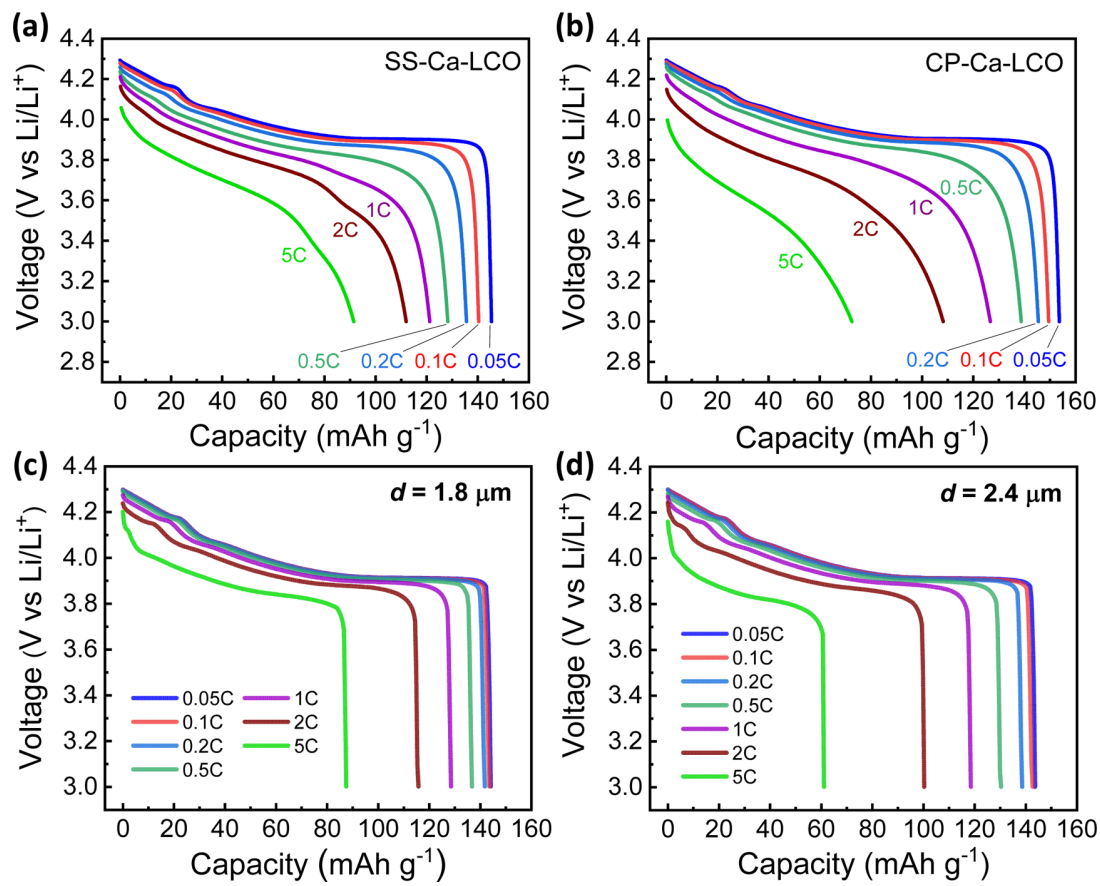

Fig. 4. Experimental discharge profile of (a) SS-Ca-LCO and (b) CP-Ca-LCO compared with simulated discharge profiles with particle diameter of (c) $1.8 \mu \mathrm{m}$ and (d) $2.4 \mu \mathrm{m}$.

with the smaller particle size exhibited higher average voltage and specific capacity compared to $\mathrm{CP}$ Ca-LCO, which had a larger particle size. The capacity was mainly determined by the time constant for the diffusion of $\mathrm{Li}^{+}$into the center of the particles. As was discussed in the previous paragraph, SS-Ca-LCO with a smaller time constant could exhibit a larger capacity at high rates compared to CP-Ca-LCO. On the other hand, the average voltage was reduced by ohmic, charge transfer, and concentration polarization. Among them, the ohmic polarization could be approximated to be constant because it was mainly owing to the electrolyte resistance, being a function of the separator's thickness. Smaller particle size contributed to reducing the overpotential in the following two mechanisms: (1) the increase in the specific surface area reduced the current density and hence, the charge transfer overpotential; (2) the decrease in the length of solid-state $\mathrm{Li}^{+}$diffusion reduced the concentration overpotential.

To investigate the influence of the particle size on the electrochemical performance, a numerical simulation was conducted using a single particle model (SPM) of $\mathrm{LCO}$ at $0.05 \mathrm{C}, 0.1 \mathrm{C}, 0.2 \mathrm{C}, 0.5 \mathrm{C}, 1 \mathrm{C}, 2 \mathrm{C}$, and 5C (Fig. S4). Figs. 4(c) and 4(d) simulate the voltage profiles for particle sizes (i.e., diameter) of 1.8 and $2.4 \mu \mathrm{m}$, respectively. The particle sizes were selected to match the simulated voltage profiles with the experimental data. The experimental particle diameter was 1.5 and $3.8 \mu \mathrm{m}$ for SS-Ca-LCO and $\mathrm{CP}-\mathrm{Ca}-\mathrm{LCO}$, respectively; and the discrepancies in the diameter may be due to the uncertainties in determining the average particle size of CP-Ca-LCO with the broad distribution of the particle size. Nevertheless, the SPM simulation provides useful understanding on the rate capability by different particle size. These observations clearly reveal that the rate capability was sensitive to the particle size of the cathode materials. The significant differences between the average voltage for the simulated and experimental profiles could be attributed to the fact that the experimental data was measured for the composite electrodes, whereas the simulation assumed single particle for clarity and simplicity.

\section{Conclusions}

Average particle size and homogeneity of the dop- 
ant closely depended on the synthetic routes of $\mathrm{Ca}$ doped $\mathrm{LiCoO}_{2}$ cathode materials. Compared with the co-precipitation, the solid-state synthesis resulted in a smaller particle size and better homogeneity of $\mathrm{Ca}^{2+}$ dopant, which positively influenced the rate and cycle performance. The better cycle performance of Ca-doped $\mathrm{LiCoO}_{2}$ from solid-state synthesis was attributed to the enhanced structural stability; which was owing to an effective doping and less severe structural damage in accordance with the alleviated concentration gradient in the smaller particles. The reduced particle size effectively decreased the length for solid-state diffusion, which enabled better rate capability compared to the Ca-doped $\mathrm{LiCoO}_{2}$ from co-precipitation. The positive role of the particle size reduction in enhancing the rate capability was also evidenced by electrochemical simulations based on a single particle model of $\mathrm{LiCoO}_{2}$ cathodes. Thus, this work established the intertwined effects of particle size and doping on the electrochemical performance of cathode materials for Li-ion batteries.

\section{Supporting Information}

Additional elemental mapping and electrochemical simulation. Supporting Information is available at https://doi.org/10.33961/jecst.2020.00899

\section{Acknowledgement}

This work was supported by a 2-year research grant of Pusan National University.

\section{References}

1. D. Larcher and J.-M. Tarascon, Nat. Chem., 2015, 7(1), 19.

2. T.H. Kim, J.S. Park, S.K. Chang, S. Choi, J.H. Ryu and H.K. Song, Adv. Energy Mater, 2012, 2(7), 860-872.

3. N. Nitta, F. Wu, J.T. Lee and G. Yushin, Mater. Today, 2015, 18(5), 252-264.

4. B. Xu, D. Qian, Z. Wang and Y.S. Meng, Mater. Sci. Eng., $R$, 2012, 73(5-6), 51-65.

5. K. Mizushima, P. Jones, P. Wiseman and J.B. Goodenough, Mater. Res. Bull., 1980, 15, 783-789.

6. S. Kalluri, M. Yoon, M. Jo, S. Park, S. Myeong, J. Kim, S.X. Dou, Z. Guo and J. Cho, Adv. Energy Mater., 2017, 7(1), 1601507

7. J. Qian, L. Liu, J. Yang, S. Li, X. Wang, H.L. Zhuang and Y. Lu, Nat. Commun., 2018, 9, 4918.

8. F. Schipper, E.M. Erickson, C. Erk, J.-Y. Shin, F.F.
Chesneau and D. Aurbach, J. Electrochem. Soc., 2017, 164(1), A6220-A6228.

9. J. Dunn, L. Gaines, J. Kelly, C. James and K. Gallagher, Energy Environ. Sci., 2015, 8(1), 158-168.

10. T. Kim, W. Song, D.-Y. Son, L.K. Ono and Y. Qi, J. Mater. Chem. A, 2019, 7(7), 2942-2964.

11. Q. Liu, X. Su, D. Lei, Y. Qin, J. Wen, F. Guo, Y.A. Wu, Y. Rong, R. Kou, X. Xiao, F. Aguesse, J. Bareño, Y. Ren, W. Lu and Y. Li, Nat. Energy, 2018, 3(11), 936-943.

12. X. Lu, Y. Sun, Z. Jian, X. He, L. Gu, Y.-S. Hu, H. Li, Z. Wang, W. Chen, X. Duan, L. Chen, J. Maier, S. Tsukimoto and Y. Ikuhara, Nano Lett., 2012, 12(12), 6192-6197.

13. X.-Y. Qiu, Q.-C. Zhuang, Q.-Q. Zhang, R. Cao, P.-Z. Ying, Y.-H. Qiang and S.-G. Sun, Phys. Chem. Chem. Phys., 2012, 14(8), 2617-2630.

14. M.D. Radin, S. Hy, M. Sina, C. Fang, H. Liu, J. Vinckeviciute, M. Zhang, M.S. Whittingham, Y.S. Meng and A. Van der Ven, Adv. Energy Mater, 2017, 7(20), 1602888 .

15. A. Van der Ven, M. Aydinol, G. Ceder, G. Kresse and J. Hafner, Phys. Rev. B, 1998, 58(6), 2975.

16. H. Xia, L. Lu, Y. Meng and G. Ceder, J. Electrochem. Soc., 2007, 154(4), A337-A342.

17. A. Yano, M. Shikano, A. Ueda, H. Sakaebe and Z. Ogumi, J. Electrochem. Soc., 2017, 164(1), A6116A6122.

18. M. Mladenov, R. Stoyanova, E. Zhecheva and S. Vassilev, Electrochem. Commun., 2001, 3(8), 410-416.

19. L. Wang, B. Chen, J. Ma, G. Cui and L. Chen, Chem. Soc. Rev, 2018, 47(17), 6505-6602.

20. C. Pouillerie, L. Croguennec, P. Biensan, P. Willmann and C. Delmas, J. Electrochem. Soc., 2000, 147, 20612069.

21. H. Li, P. Zhou, F. Liu, H. Li, F. Cheng and J. Chen, Chem. Sci., 2019, 10(5), 1374-1379.

22. H. Tukamoto and A. West, J. Electrochem. Soc., 1997, 144(9), 3164-3168.

23. M. Jo, Y.-S. Hong, J. Choo and J. Cho, J. Electrochem. Soc., 2009, 156(6), A430-A434.

24. M. Okubo, E. Hosono, J. Kim, M. Enomoto, N. Kojima, T. Kudo, H. Zhou and I. Honma, J. Am. Chem. Soc., 2007, 129(23), 7444-7452.

25. C. Mi, X. Zhao, G. Cao and J. Tu, J. Electrochem. Soc., 2005, 152(3), A483-A487.

26. R. Malik, D. Burch, M. Bazant and G. Ceder, Nano Lett., 2010, 10(10), 4123-4127.

27. H. Zheng, J. Li, X. Song, G. Liu and V.S. Battaglia, Electrochim. Acta, 2012, 71, 258-265.

28. A. Gusev and A. Kurlov, Nanotechnology, 2008, 19(26), 265302.

29. M. Salari, M. Rezaee and P. Marashi, J. Nano Res-SW, 2009, 6, 15-21.

30. M. Stein, IV, C.-F. Chen, M. Mullings, D. Jaime, A. Zaleski, P.P. Mukherjee and C.P. Rhodes, J. Electrochem. Energy, 2016, 13(3). 
31. T. Pan, J. Alvarado, J. Zhu, Y. Yue, H.L. Xin, D. Nordlund, F. Lin and M.M. Doeff, J. Electrochem. Soc., 2019, 166(10), A1964-A1971.

32. K.-M. Nam, H.-J. Kim, D.-H. Kang, Y.-S. Kim and S.-W. Song, Green Chem., 2015, 17(2), 1127-1135.
33. P. Barai, Z. Feng, H. Kondo and V. Srinivasan, J. Phys. Chem. B, 2019, 123(15), 3291-3303.

34. G. Ting-Kuo Fey, V. Subramanian and J.-G. Chen, Electrochem. Commun., 2001, 3(5), 234-238. 